\title{
Avaliação do nível de conhecimento das prescrições na Atenção Primária à Saúde
}

\author{
Assessment of the level of knowledge of prescriptions on Primary Health Care \\ Evaluación del nível de conocimento de las recetas em Atención Primaria de Salud
}

Marcelo da Silva

ORCID: https://orcid.org/0000-0003-4509-8294 Christus Faculdade do Piauí, Brasil E-mail: marceloenf21@gmail.com

Ana Paula Melo Oliveira

ORCID: https://orcid.org/0000-0003-3328-9624 Christus Faculdade do Piauí, Brasil

E-mail: apmeloenfer@gmail.com

Kayco Damasceno Pereira

ORCID: https://orcid.org/0000-0002-3112-1193 Christus Faculdade do Piauí, Brasil

E-mail: kaycopereir@gmail.com

Karoline Maria Mendes Amaral ORCID: https://orcid.org/0000-0003-2187-202X Christus Faculdade do Piauí, Brasil

E-mail: karolineamaral308@gmail.com

Marcos Roberto Nascimento Sousa ORCID: https://orcid.org/0000-0003-1634-5276 Christus Faculdade do Piauí, Brasil

E-mail: marcosrobertoenfpi@gmail.com

Sabrina Sousa Barros

ORCID: https://orcid.org/0000-0003-4517-0401

Christus Faculdade do Piauí, Brasil

E-mail: sabrinabarros1901@gmail.com

Evaldo Sales Leal

ORCID: https://orcid.org/0000-0002-1424-9048

Christus Faculdade do Piauí, Brasil

E-mail: evaldosleal@hotmail.com

Carliane Maria de Araújo Souza

ORCID: https://orcid.org/ 0000-0002-8196-0008

Christus Faculdade do Piauí, Brasil

E-mail:kku_ka@hotmail.com

Sabrina Beatriz Mendes Nery

ORCID: https://orcid.org/0000-0002-8254-0152

Christus Faculdade do Piauí, Brasil

E-mail: sabrinaanery2019@gmail.com

Sara Samara Ferreira de Araújo

ORCID: https://orcid.org/0000-0003-2638-1094

Christus Faculdade do Piauí, Brasil

E-mail: sarasam231@gmail.com

Anderson Pereira Freitas

ORCID: https://orcid.org/0000-0003-2899-6148

Christus Faculdade do Piauí, Brasil

E-mail: andersonft10tb@gmail.com

Maria Vitória Frota Magalhães

ORCID: https://orcid.org/0000-0001-7597-0616

Christus Faculdade do Piauí, Brasil

E-mail: vitoriafrotam@gmail.com

Gabrielly Silva Ramos

ORCID: https://orcid.org/0000-0003-0767-1352

Christus Faculdade do Piauí, Brasil

E-mail: gabiramos2304@gmail.com

Amanda Silva do Nascimento

ORCID: https://orcid.org/0000-0002-5518-4922

Christus Faculdade do Piauí, Brasil

E-mail: amandanascimento0613@gmail.com

Iasmim Escórcio de Brito Melo

ORCID: https://orcid.org/0000-0002-5984-0309

Christus Faculdade do Piauí, Brasil

E-mail: Iasmimedb@gmail.com 


\begin{abstract}
Resumo
A prescrição medicamentosa é uma ferramenta fundamental que pode evitar o uso irracional de medicamentos minimizando os riscos e favorecendo os seus benefícios, portanto, deve ser compreendida pelo usuário para que os seus objetivos sejam alcançados.Com o intuito de responder à problemática desta pesquisa, objetivou-se avaliar o nível de conhecimento dos usuários da Atenção Básica $(\mathrm{AB})$ sobre as suas prescrições, assim como traçar o perfil sociodemográfico da população, caracterizar e discutir os principais fatores que influenciam com esse problema. $\mathrm{O}$ estudo trata-se de uma pesquisa de campo com abordagem quantitativa realizada no contexto da $\mathrm{AB}$. Os dados foram coletados através de uma entrevista realizada com 210 participantes que possuíam uma prescrição medicamentosa emitida por um enfermeiro, médico ou cirurgião dentista. A pesquisa teve início após aprovação pelo Comitê de Ética, assegurando os direitos dos participantes de acordo com os aspectos éticos e legais. Os resultados evidenciaram que a população estudada é em sua maioria do sexo feminino, idosa e de baixa renda. Em relação à compreensão, foi possível observar uma grande deficiência no conhecimento sobre possíveis interações medicamentosas e reações adversas, seguido do tempo do tratamento e nome do medicamento, revelando os ricos que os usuários estão expostos devido ao entendimento ineficaz das suas prescrições. Desta forma, conclui-se que há a necessidade de preparar os profissionais prescritores a realizarem uma prescrição racional, instruindo de forma mais efetiva os usuários sobre o seguimento da sua terapia medicamentosa que possibilite minimizar os seus riscos e promover o Uso Racional de Medicamentos.
\end{abstract}

Palavras-chave Compreensão; Conhecimento do paciente sobre a medicação; Prescrições de medicamentos; Atenção primária à saúde.

\begin{abstract}
The prescription drug is a fundamental tool that can avoid the irrational use of medicines minimizing the risks and favoring its benefits, therefore, it must be understood by the user so that its objectives are reached. - to assess the level of knowledge of Primary Health Care (PHC) users about their prescriptions, as well as to outline the population's socio-demographic profile, to characterize and discuss the main factors that influence this problem. The study is a field research with a quantitative approach carried out in the context of PHC. Data were collected through an interview with 210 participants who had a prescription issued by a nurse, doctor or dentist. The research started after approval by the Ethics Committee, ensuring the participants' rights in accordance with ethical and legal aspects. The results showed that the population studied is mostly female, elderly and low income. Regarding understanding, it was possible to observe a great deficiency in the knowledge about possible drug interactions and adverse reactions, followed by the time of treatment and name of the drug, revealing the rich that users are exposed due to the ineffective understanding of their prescriptions. Thus, it is concluded that there is a need to prepare the prescribing professionals to carry out a rational prescription, instructing users more effectively on the follow-up of their drug therapy, which makes it possible to minimize their risks and promote the Rational Use of Medicines.
\end{abstract}

Keywords: Comprehension; Patient medication knowledge; Drug prescriptions; Primary health care.

\title{
Resumen
}

El medicamento de prescripción es una herramienta fundamental que puede evitar el uso irracional de medicamentos minimizando los riesgos y favoreciendo sus beneficios, por lo tanto, debe ser entendido por el usuario para que se alcancen sus objetivos.- Para dar respuesta a la problemática de esta investigación, el objetivo fue evaluar el nivel de conocimiento de los usuarios de Atención Primaria (AP) sobre sus prescripciones, así como delinear el perfil sociodemográfico de la población, caracterizar y discutir los principales factores que influyen influencia con este problema. El estudio es una investigación de campo con un enfoque cuantitativo realizada en el contexto de AP. Los datos se recopilaron a través de una entrevista con 210 participantes que tenían una receta emitida por una enfermera, médico o dentista. La investigación se inició después de la aprobación del Comité de Ética, garantizando los derechos de los participantes de acuerdo con los aspectos éticos y legales. Los resultados mostraron que la población estudiada es mayoritariamente femenina, de edad avanzada y de bajos ingresos. En cuanto al entendimiento, se pudo observar una gran deficiencia en el conocimiento sobre posibles interacciones farmacológicas y reacciones adversas, seguido del tiempo de tratamiento y nombre del fármaco, revelando los ricos a los que están expuestos los usuarios debido a la comprensión ineficaz de sus prescripciones. Así, se concluye que existe la necesidad de preparar a los profesionales prescriptores para que realicen una prescripción racional, instruyendo de manera más eficaz a los usuarios en el seguimiento de su farmacoterapia, que permita minimizar sus riesgos y promover el Uso Racional Medicamentos.

Palabras clave: Comprensión; Conocimiento de la medicación por el paciente; Prescripciones de medicamentos; Atención primaria de salud.

\section{Introdução}

Os medicamentos são importantes mecanismos terapêuticos e um bem significativo para a saúde, sendo responsável pelo importante aumento da expectativa e qualidade de vida dos indivíduos. A evolução da terapêutica farmacológica tem sido visível desde o surgimento dos primeiros medicamentos para o controle de infecções entre 1930 e 1940, que contribuíram 
fortemente com a redução da morbimortalidade ao longo do século passado. A partir daí os medicamentos começaram a ser vistos como elementos complexos, deixando de ser apenas um instrumento de intervenção terapêutica (Arrais et al., 2005; Banõs-Diéz \& Farré-Albaladejo, 2002).

Os potenciais benefícios do uso de medicamentos, em geral, são bem conhecidos, porém, mesmo na época do surgimento dos primeiros fármacos, já era conhecida a capacidade dos medicamentos de provocar efeitos adversos no organismo. Desta forma, em 1985 surge o conceito de promoção do Uso Racional de Medicamentos (URM), proposto pela Organização Mundial da Saúde (OMS), que traz a informação de que o paciente deve receber a medicação correta, na dose adequada e em um determinado período de tempo, considerando também, o custo individual e comunitário da droga. Tendo em vista estas informações, os profissionais prescritores necessitam permanecer cautelosos às inúmeras questões ligadas ao tratamento farmacológico do cliente, analisando se na realidade, determinada medicação está adequada para o doente, se é segura, efetiva e se há aceitação do paciente à terapêutica proposta (Melo et al., 2006; Who, 1986; Brasil, 2019a).

Sabendo disso, a prescrição torna-se um importante instrumento neste contexto, pois contribui com a intervenção de agravos, favorecendo que as vantagens intrínsecas da utilização dos medicamentos sejam maiores que os riscos, sendo que a prescrição é uma forma de determinar qual intervenção medicamentosa deve ser feita pelo paciente, orientando de forma escrita, todas as informações essenciais pertinentes ao tratamento em questão, além de ser um documento necessário para a dispensação e aquisição de alguns fármacos, evitando a automedicação e aumentando a segurança do paciente no processo terapêutico (Brasil, 2001; Sharif et al., 2008).

Grande parte das inúmeras prescrições realizadas no Sistema Único de Saúde (SUS), não apresentam a disposição dos requisitos legais e técnicos exigidos e fundamentais para pôr em prática o uso racional e dispensação efetiva dos medicamentos. Pela ausência de informações importantes negligenciadas pelos profissionais durante a consulta, há muitos pacientes que sentem dificuldades para compreender o tratamento indicado, favorecendo a incerteza e descontinuidade da intervenção terapêutica, causando ineficácia do tratamento ou danos à saúde (Lyra Junior et al., 2004; Higashi et al., 2004; Portela et al., 2010).

O uso incorreto de fármacos não resulta apenas em riscos à saúde do indivíduo ou perdas individuais, mas também representa um importante dano de ordem econômica para o poder público. Estima-se que a metade da população que faz uso de medicamentos os utilizam de maneira incorreta, tornando este, um problema de saúde pública, contribuindo com o gasto elevado dos hospitais decorrente das despesas provocadas pela intervenção de complicações originadas por este uso indevido; fazendo com que um terço das internações hospitalares no Brasil sejam resultantes desta prática inadequada (Aquino, 2008; Portela et al., 2010; Opas, 2005; Opas, 2007).

O baixo nível de conhecimento da população acerca do seguimento correto do tratamento prescrito pode resultar em grandes malefícios, tanto para a saúde do indivíduo, quanto para o patrimônio econômico próprio e coletivo, uma vez que o uso irracional de medicamentos pode ocasionar alterações no organismo provocando um efeito danoso, acarretando no adoecimento das pessoas saudáveis e piorando o quadro das já debilitadas. Desta forma, o problema central deste estudo pode ser expresso na seguinte indagação: qual o nível de conhecimento dos usuários acerca das prescrições medicamentosas na Atenção Primária à Saúde?

Com base nisso, o desenvolvimento da presente pesquisa resulta de uma preocupação com o amplo uso de medicamentos, falta de orientações necessárias e a forma como os prescritores abordam as informações presentes nas prescrições realizadas. A falta de orientação por profissionais, quanto ao uso da medicação implica em resultados inesperados, podendo colocar em risco a qualidade de vida e bem-estar dos indivíduos, corrobora com a disseminação de informações falsas e contribui com o uso indiscriminado de medicamentos, uma vez que, existem diversos grupos populacionais com diferentes 
necessidades, características particulares e até mesmo linguagens próprias que necessitam de uma abordagem de acordo com suas peculiaridades para que haja uma comunicação eficaz no que se refere às informações repassadas nas prescrições.

É comum nos depararmos com situações em que os indivíduos sem conhecimento técnico, idosos e/ou analfabetos, sentem dificuldades ou desconhecem o seguimento de um tratamento favorecendo uma interação medicamentosa e aumentando os riscos de intoxicação. O desconhecimento acerca do armazenamento adequado e dos prazos de validade dos medicamentos também oferecem riscos à saúde dos indivíduos. Frente a todos os problemas que podem ser gerados pela má interpretação e qualidade das informações presentes nas prescrições, assim como a escassez de estudos que abordem o tema, propõe-se o presente projeto de pesquisa, visando ampliar o conhecimento acerca do tema e a melhoria da qualidade da assistência prestada no âmbito da Atenção Primária à Saúde e autocuidado.

Com o intuito de responder à problemática deste estudo, objetivou-se primariamente avaliar o nível de conhecimento dos usuários acerca das prescrições medicamentosas na Atenção Primária a Saúde, e, secundariamente, objetivou-se caracterizar a população da pesquisa de acordo com o nível de compreensão, assim como, identificar os motivos que influenciam na compreensão das prescrições na Atenção Primária à Saúde, traçar o perfil sociodemográfico da população inclusa no estudo, avaliar as consequências provocadas pela falta de compreensão acerca das prescrições medicamentosas, e por fim, discutir sobre as principais dificuldades enfrentadas pelos pacientes frente às prescrições medicamentosas na Atenção Primária à Saúde.

\section{Metodologia}

\subsection{Delineamento e local do estudo}

A coleta de dados teve início mediante a aprovação pelo Comitê de Ética do Centro Universitário da Faculdade de Saúde, Ciências Humanas e Tecnológicas do Piauí - UNINOVAFAPI, sob parecer de $\mathrm{n}^{\circ}$ 4.419.693, de acordo com a Resolução n 466/12, n 510/16 e n 580/18 do Conselho Nacional de Saúde (CNS), que indicam sobre as pesquisas científicas que envolvem seres humanos, trazendo o dever do pesquisador de obedecer aos princípios éticos e científicos equivalentes, respeitando os envolvidos em sua vulnerabilidade, dignidade e autonomia.

Este estudo trata-se de uma pesquisa de campo do tipo transversal, abordagem quantitativa, objetivo descritivo e natureza aplicada. O estudo foi feito no município de Piripiri que possui área territorial de aproximadamente $1.409,250 \mathrm{~km}^{2} \mathrm{e}$ uma população de 63.742 habitantes, fica localizado na região denominada território dos Cocais que abrange 22 municípios do norte do Piauí (Brasil, 2019b; Brasil, 2015).

\subsection{População do estudo e casuística}

A pesquisa foi realizada no contexto da Atenção primária à Saúde que é composta por 29 unidades de Estratégia de Saúde da Família de diferentes bairros da zona urbana e localidades rurais do município; sendo aplicada na totalidade do número de unidades em funcionamento, que por sua vez, estão aptas para atenderem aos 63 mil habitantes do município.

Sabendo disso, a amostra prevista da população estudada foi inicialmente de 388 usuários, calculada em amostra finita e obedecendo um erro amostral de 5\% e intervalo de confiança de 95\%, na calculadora on-line Survey Monkey. Ressalta-se que seriam escolhidos aleatoriamente 14 participantes em cada ESF. No entanto, por ocasião da Pandemia de SaRS-Cov-2 (COVID-19), a demanda por atendimentos diminuiu consideravelmente, comprometendo o total de participantes, que ao fim da pesquisa, totalizou 210 participantes.

Foram incluídos no estudo, os usuários da APS com idade igual ou maior que 18 anos; que utilizaram o serviço de saúde ou responsável por um menor de idade ou outro dependente que o utilizou, sendo considerados os serviços médicos, de enfermagem e odontológicos; que tinham pelo menos um medicamento prescrito durante o atendimento ou ser responsável 
pela medicação de um menor de idade ou outro dependente que tenha sido prescrito um ou mais medicamento; e que assinaram e demonstraram estar de acordo com o Termo de Consentimento Livre e Esclarecido (TCLE).

\subsection{Coleta de dados}

O instrumento de coleta de dados para quantificar o nível de conhecimento dos usuários sobre a prescrição medicamentosa, que foi usado nesta investigação, foi desenvolvido e testado por Fröhlich et al. (2010) e utilizado em outras pesquisas como a realizada por Zanetti (2016). O instrumento contempla perguntas objetivas relacionadas ao nome, função, posologia, precauções e possíveis efeitos indesejados dos medicamentos prescritos e se adequa com os objetivos do presente projeto de pesquisa.

Os dados sociodemográficos foram colhidos através da ficha de cadastro contendo a identificação do pesquisador e participante; local, data e horário da entrevista, além dos indicadores de idade, sexo, cor/etnia, grau de escolaridade, renda familiar, ocupações e estado civil.

\section{Resultados e Discussão}

Os resultados expostos a seguir, referem-se aos dados coletados de um total de 210 participantes que aceitaram contribuir com a pesquisa respondendo a entrevista e que se adequaram aos critérios de inclusão. Foram abordados 302 indivíduos, dos quais 92 se recusaram a participar e/ou não estavam elegíveis de acordo com as normas de participação.

As entrevistas realizadas duraram em média 15 minutos sofrendo influência da idade dos indivíduos e dúvidas dos usuários que foram sendo esclarecidas durante o tempo da entrevista. Dos que se negaram a participar, os principais motivos foram o desinteresse em participar do estudo e a falta de tempo justificada pela demora no atendimento.

A exposição e a discussão dos dados coletados estão descritos em dois eixos, o primeiro trata-se da descrição do perfil sociodemográfico dos participantes e o segundo aborda sobre o conhecimento dos usuários acerca das suas prescrições no âmbito da Atenção Primária à Saúde no município estudado.

\subsection{Perfil sociodemográfico e econômico dos usuários da Atenção Primária à Saúde inclusos na pesquisa}

Do número total de participantes inclusos, 70,95\% (n=149) são do sexo feminino e 29,05\% ( $\mathrm{n}=61)$, do sexo masculino. Esse resultado pode estar relacionado com a maior participação das mulheres na busca por atendimento na APS que equivale a aproximadamente $76 \%$ dos usuários, segundo estudo nacional realizado por Guibu e colaboradores (2017). Com relação à faixa etária, prevaleceu a população idosa (>60 anos) com 51,43\% dos entrevistados, seguido da população de meia idade (40-59 anos) com 25,71\% e jovens adultos (18-39 anos) com 22,86\%. Esses valores foram diferentes dos resultados descritos por Ascef et al. (2017), onde apontou que a maioria dos usuários da $A B$ são de jovens adultos (39,7\%) enquanto a menor porcentagem é composta pelos idosos (23,0\%), e a população de meia idade equivale a 37,3\% dos usuários.

Assim como descrito por Costa et al. (2017), nesta pesquisa também foi possível observar uma crescente prevalência do uso de medicamentos conforme o passar da idade, o número médio de medicação em uso teve um aumento considerável de acordo com a faixa etária, sendo que indivíduos mais jovens tiveram um valor aproximado de 1,2 medicamentos em uso e a população idosa faziam uso de até 4 medicamentos. Isso tem relação com as alterações progressivas do envelhecimento e o surgimento de doenças crônicas que necessitam de um tratamento (Ascef et al., 2017; Costa et al., 2017).

Considerando a cor da pele, foi possível observar uma prevalência de indivíduos autodeclarados pardos $(49,19 \%)$ e pretos $(30 \%)$, seguido respectivamente por brancos $(20,95 \%)$, indígenas $(1,9 \%)$ e amarelos $(0,95 \%)$. Quando comparados a média nacional, esses resultados divergem em alguns aspectos, pois apenas o número de pardos é equiparável (50,5\%), 
havendo uma variação significativa dos demais grupos, onde a população branca vem em segunda colocação com $39,7 \%$, seguida respectivamente pelos pretos (7,8\%), amarela (1,5\%) e indígena (0,2\%) (Guibu et al., 2017).

As estatísticas quando comparadas com a região Nordeste os valores sofrem algumas mudanças em relação à média do Brasil, mas a divergência das porcentagens com a média do município ainda se mantém. Levando em consideração apenas à média nordestina, os pardos equivalem aproximadamente a $66 \%$, seguido respectivamente da população branca $(25,7 \%)$, preta $(7,8 \%)$, amarela $(0,3 \%)$ e indígena $(0,2 \%)$. Neste caso, assim como no Brasil, o Nordeste possui uma população de amarelos maior que indígenas, prevalência diferente do encontrado no município. Esse fato pode ser consequência da existência de uma pequena comunidade indígena na região estudada (Guibu et al., 2017).

A Tabela 1 apresenta o número de usuários entrevistados nos serviços da APS de acordo com as características sociodemográficas nas variáveis de sexo, idade, cor da pele, nível de escolaridade e estado civil.

Tabela 1 - Características sociodemográficas obtidas através da ficha de identificação e de coleta de dados sociodemográficos dos usuários da Atenção Primária à Saúde inclusos na pesquisa. Piripiri-PI, 2020.

\begin{tabular}{|c|c|c|}
\hline VARIÁVEIS SOCIODEMOGRÁFICAS & $\mathbf{N}^{\circ}$ & $\%$ \\
\hline $\mathrm{N}^{\circ}$ de mulheres entrevistadas & 149 & $70,95 \%$ \\
\hline $\mathrm{N}^{\circ}$ de homens entrevistados & 61 & $29,05 \%$ \\
\hline \multicolumn{3}{|l|}{ Faixa etária (anos) } \\
\hline $18-39$ & 48 & $22,86 \%$ \\
\hline $40-59$ & 54 & $25,71 \%$ \\
\hline $60 \mathrm{ou}+$ & 108 & $51,43 \%$ \\
\hline \multicolumn{3}{|l|}{ Cor da pele } \\
\hline Parda & 97 & $46,19 \%$ \\
\hline Preta & 63 & $30,00 \%$ \\
\hline Branca & 44 & $20,95 \%$ \\
\hline Indígena & 4 & $1,90 \%$ \\
\hline Amarela & 2 & $0,95 \%$ \\
\hline \multicolumn{3}{|l|}{ Nível de escolaridade } \\
\hline Nunca frequentou a escola & 32 & $15,24 \%$ \\
\hline Ensino Fundamental Incompleto & 46 & $21,90 \%$ \\
\hline Ensino Fundamental Completo & 20 & $9,52 \%$ \\
\hline Ensino Médio Incompleto & 19 & $9,05 \%$ \\
\hline Ensino Médio Completo & 53 & $25,24 \%$ \\
\hline Ensino Superior Incompleto & 22 & $10,48 \%$ \\
\hline Ensino Superior Completo & 18 & $8,57 \%$ \\
\hline \multicolumn{3}{|l|}{ Sabe ler e escrever / Alfabetizado } \\
\hline Sim & 141 & $67,14 \%$ \\
\hline Não & 69 & $32,86 \%$ \\
\hline \multicolumn{3}{|l|}{ Estado civil } \\
\hline Casado & 54 & $25,71 \%$ \\
\hline União estável & 74 & $35,24 \%$ \\
\hline Solteiro & 48 & $22,86 \%$ \\
\hline Viúvo & 19 & $9,05 \%$ \\
\hline Divorciado & 15 & $7,14 \%$ \\
\hline
\end{tabular}

Fonte: Autores (2020).

Observando o nível de escolaridade (Tabela 1), dos 210 usuários inclusos, apenas 8,57\% possuem ensino superior, $35,71 \%$ concluíram o ensino médio enquanto $15,24 \%$ nunca frequentaram a escola. A porcentagem de indivíduos que possuem 
ensino superior foi elevada, quando comparada com a média brasileira. De acordo com estudo realizado no ano de 2017, que avaliou as características dos usuários da APS do SUS nas cinco regiões geopolíticas do país, apenas 3,1\% da população usuária da $\mathrm{AB}$ possuem alguma formação de nível superior (Ascef et al., 2017).

Apesar do número de usuários pesquisados que possuem ensino superior seja acima da média nacional, também há um elevado número de pessoas analfabetas $(32,86 \%)$ quando comparado às estatísticas do país, das regiões Sul, Sudeste, Norte e também Nordeste, que possui o maior índice de analfabetismo entre as cinco regiões. O número dos participantes que possuem ensino médio foi a única variável que coincidiu com o valor nacional, onde equivale a 25,6\% da população que faz uso da APS (Guibu et al., 2017; Ascef et al., 2017).

De acordo com o estado civil (Tabela 1), a maioria relatou estar em uma união estável $(35,24 \%$ ), seguido respectivamente pelos casados $(25,71 \%)$, solteiros $(22,86 \%)$, viúvos $(9,05 \%)$ e divorciados $(7,14 \%)$. Apesar da união estável se destacar nesse caso, em estudos nacionais citados anteriormente, o estado civil predominante no Brasil e Nordeste é constituído pela população casada, enquanto a união estável equivale apenas a 21,3\% no Nordeste e $16 \%$ no Brasil. A taxa de solteiros é equivalente à média nordestina e brasileira sendo de $21,4 \%$ e $21,8 \%$, respectivamente, assim como os divorciados que representam 5,3\% e 6,7\% das populações. Os usuários viúvos, no país, podem variar de 6\% a 7,5\% aproximadamente (Guibu et al., 2017).

A seguir, foi traçado o panorama da relação dos usuários da $\mathrm{AB}$ que foram entrevistados e suas condições econômicas de acordo com as variáveis de renda familiar, ocupação, quantidade de pessoas que contribuem na renda da família e seus dependentes. Como descrito por Ascef et al. (2017), a maioria dos usuários da APS são considerados de baixa renda. Sabendo disso, pode-se observar que os resultados desta pesquisa corroboram com o descrito, sendo que dentre os participantes entrevistados, aproximadamente 70,95\% ( $\mathrm{n}=149)$ podem ser classificados como de baixa renda, desses, 16,11\% ( $\mathrm{n}=25)$ estão em situação de vulnerabilidade econômica. Com relação à renda média dos demais, apenas 8,57\% (n=18) possuem renda individual próximo a um salário mínimo, como pode ser visto na figura abaixo (Figura 1). No total, a renda per capita média da população equivale a $\mathbf{R}$ 770,00, superior à média encontrada em estudo semelhante em Ribeirão Preto-SP, onde o valor foi de $\mathrm{R} \$ 663,15$ (Zanetti, 2016).

Figura 1 - Dados comparativos da renda per capita mensal dos usuários da Atenção Primária à Saúde inclusos na pesquisa. Piripiri-PI, 2020.

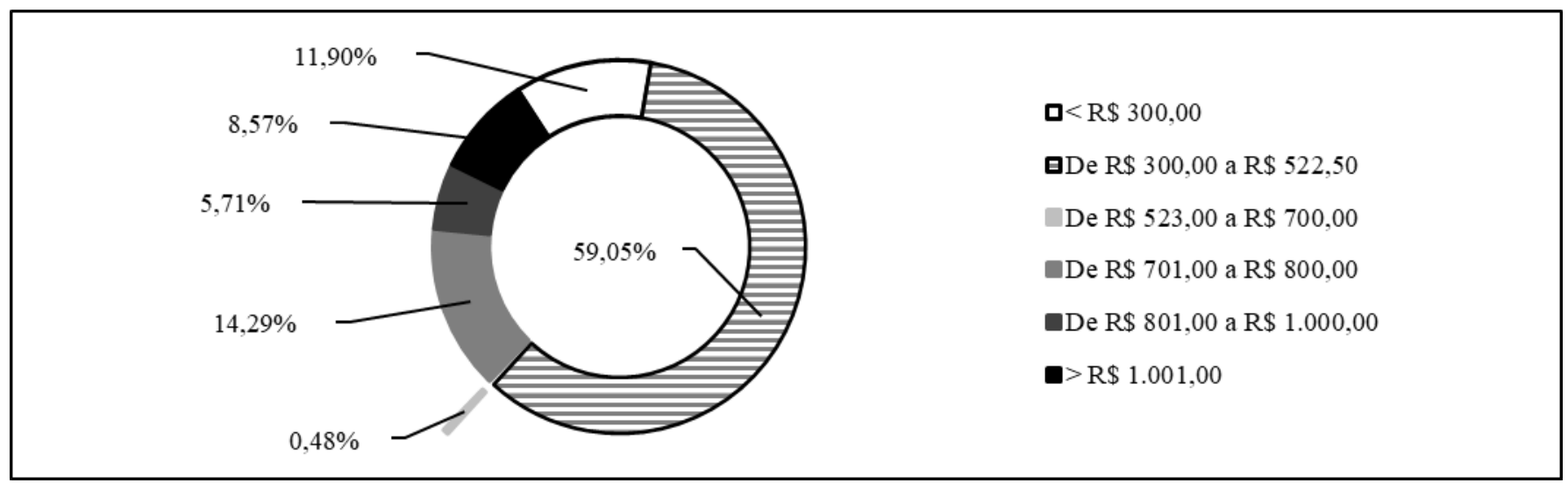

*Valor do salário mínimo no Brasil no ano de 2020: R\$ 1045,00.

**Considerando a baixa renda no Brasil no ano de 2020: renda per capita $\leq \mathrm{R} \$ 522,50$.

Fonte: Autores (2020). 
Quanto ao número de pessoas que contribuíram com a renda familiar, o número variou entre 1 a 4, com média total de $\cong 1,7$ indivíduos que contribuem com a renda mensal em famílias compostas por uma média de $\cong 3,7$ indivíduos dependentes da renda (variando entre 1 a 9 membros familiares).

Sobre os dados relativos ao tipo de ocupação das pessoas que contribuíram com a pesquisa (Tabela 2), quase a metade dos indivíduos $(48,10 \%)$ possuem renda derivada exclusivamente do trabalho e não possuem outra ocupação, porém, ao incluir a porcentagem de quem trabalha/estuda (10\%) e aqueles que trabalham e são aposentados ou pensionista $(1,43 \%)$, o número de empregados cresce para 59,52\% ( $\mathrm{n}=125)$. O grupo dos aposentados ou pensionistas é o segundo grupo mais prevalente entre os entrevistados com 22,38\% ( $n=47)$, seguido dos estudantes com 16,19\% $(n=34)$ e por último os desempregados $(13,33 \%)$.

Tabela 2 - Dados comparativos em relação à situação profissional e ocupação dos usuários da Atenção Primária à Saúde inclusos na pesquisa. Piripiri-PI, 2020.

\begin{tabular}{lcc}
\hline \multicolumn{1}{c}{ VARIÁVEIS SOCIOECONÔMICAS } & $\mathbf{N}^{\circ}$ & $\boldsymbol{\%}^{\circ}$ \\
\hline Ocupação & & $48,10 \%$ \\
Trabalha & 101 & $6,19 \%$ \\
Estudante & 13 & $20,95 \%$ \\
Aposentado ou pensionista & 44 & $13,33 \%$ \\
Desempregado & 28 & $10,00 \%$ \\
Trabalha / Estudante & 21 & $1,43 \%$ \\
Trabalha / Aposentado ou pensionista & 3 & \\
\hline
\end{tabular}

Fonte: Autores (2020).

A taxa de desemprego foi inferior ao número encontrado por Zanetti (2016), onde os desempregados/sem ocupação foram em torno de $26,2 \%$, estudantes equivale apenas a $1,7 \%$ e o grupo dos aposentados e pensionistas prevaleceu com $38,2 \%$ seguido dos trabalhadores que foi igual a $35,1 \%$ dos entrevistados. A incompatibilidade de informações pode ser explicada pelas diferenças entre as regiões estudadas e suas particularidades.

\subsection{Nível de conhecimento dos usuários da Atenção Primária à Saúde acerca das suas prescrições}

Os resultados e discussão deste tópico são derivados do questionário aplicado aos usuários que utilizam os serviços da atenção básica no município. O instrumento utilizado para a coleta de dados estruturou-se em 10 perguntas obrigatórias e 2 dependentes das respostas ao decorrer da entrevista. As perguntas foram relacionadas a compreensão das prescrições e do uso das medicações provenientes dos serviços da APS e avaliou se o participante tinha conhecimento sobre o nome do medicamento, motivo da prescrição, dose, horários e tempo de uso, modo de administração, conduta em caso de esquecimento, interações do medicamento com outros alimentos e remédios, riscos de reações adversas e quais as maiores dificuldades relacionadas à falta de informação.

A Figura 2, apresentada a seguir, traz os resultados obtidos quando se foi perguntado sobre o nome do medicamento e sua indicação terapêutica, onde 59,52\% ( $\mathrm{n}=125)$ dos participantes afirmaram saber o nome do medicamento. Apesar do número de pessoas que sabem o nome do medicamento ser superior, a porcentagem dos que desconhecem $(40,48 \%$ / n=85) é relativamente alta quando se trata de agentes químicos que quando usados de forma inadvertida e incorreta pode causar prejuízos à saúde do indivíduo e, saber o nome e para que serve é o primeiro passo para evitar erros de medicação, onde a falta de informação é uma das principais causas relacionadas aos riscos do uso de fármacos (Scripcaru et al., 2017).

Em relação ao motivo que o profissional prescreveu o medicamento, 76,19\% (n=160) sabiam para que foi indicado e $23,81 \%$ ( $n=50$ ) não sabiam o motivo da prescrição, como ilustrado no gráfico abaixo (Figura 2). Essa variável teve resultado 
equivalente na pesquisa de Fröhlich et al. (2010) que indicaram uma prevalência do conhecimento de 77,8\% dos usuários em relação à indicação terapêutica.

Figura 2 - Comparativo das respostas relacionadas ao conhecimento do nome do medicamento e motivo da prescrição dos usuários. Piripiri-PI, 2020.

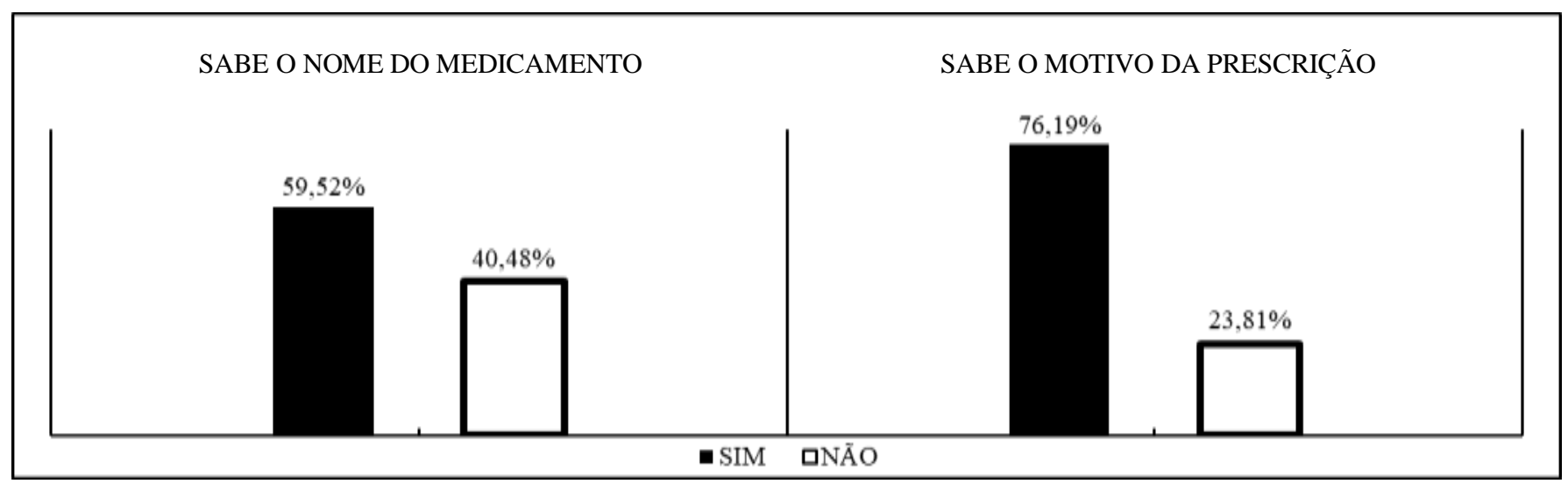

Fonte: Autores (2020).

Esse alto índice de participantes que sabiam para que o medicamento foi prescrito é resultante da relação lógica entre a medicação e a queixa que provocou a procura do usuário pelo sistema de saúde. A alta taxa de usuários que possuem doenças crônicas que fazem uso de medicamentos também influencia no valor, pois o uso prolongado do fármaco favorece esse conhecimento. A porcentagem inferior de indivíduos que sabem o nome da medicação comparado ao conhecimento da indicação terapêutica indica que 16,67\% fazem uso do medicamento sem ao menos conhecer o seu nome (Ascef et al., 2017).

A Figura 3 representa o número de pessoas que conhecem, ou não, as doses, assim como os horários de administração e tempo do tratamento prescrito. As variáveis referentes ao conhecimento da dose e os horários da administração dos medicamentos obtiveram resultados positivos, sendo que aproximadamente $70 \%$ dos participantes tem conhecimento de ambos os fatores $(\mathrm{n} \cong 147,5)$, resultado parecido ao encontrado em um estudo semelhante realizado anteriormente, onde 77,5\% dos participantes detinham do conhecimento relacionado a essa variável. O fato dos participantes terem melhor desempenho ao responderem os questionamentos relacionados aos horários e a dose, do que o próprio nome do medicamento indica que há uma maior exposição ao risco da troca da medicação nos casos de polifarmácia (Zanetti, 2016; Coradi et al., 2016).

Quanto ao conhecimento do tempo necessário para o tratamento, o valor sofre uma queda comparado com as variáveis anteriormente expostas, pois um pouco mais da metade, $56,10 \%(\mathrm{n}=118)$, sabem o período da terapia medicamentosa que, na maioria das vezes, é interrompido após o desaparecimento dos sinais e sintomas da doença fazendo com que haja uma diminuição da eficácia ou ineficiência do tratamento resultando no reaparecimento da doença (Souza et al., 2018). 
Figura 3 - Comparativo das respostas relacionadas ao conhecimento da dose, horário da medicação e tempo do tratamento dos usuários. Piripiri-PI, 2020.

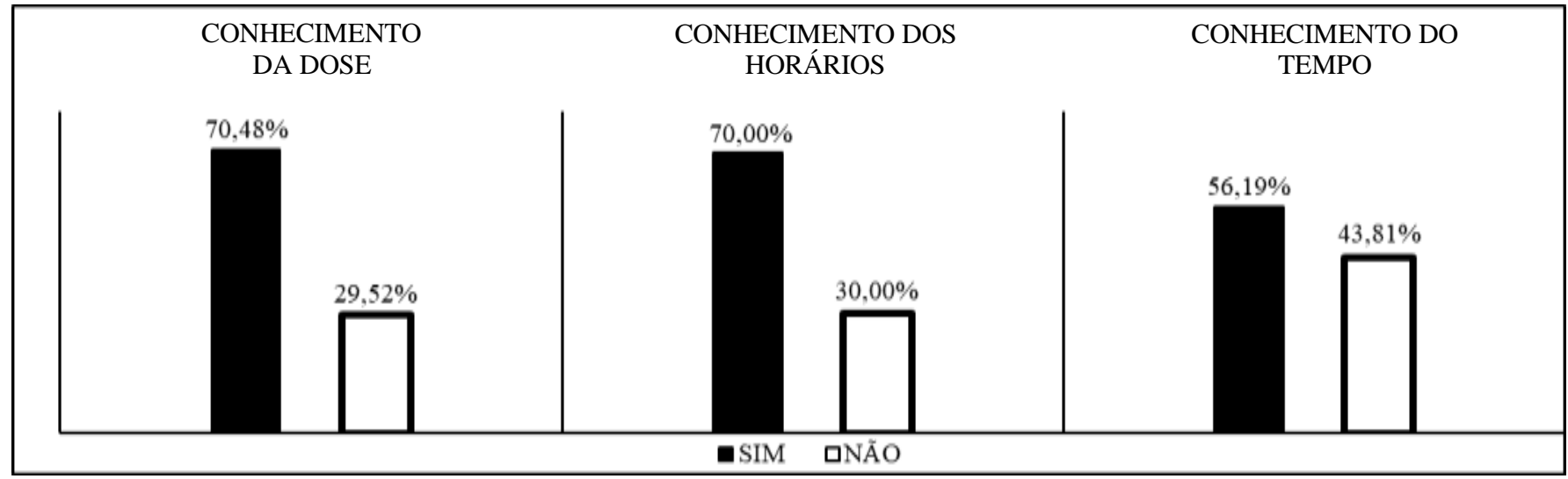

Fonte: Autores (2020).

Segundo Zanetti (2016), praticamente metade dos participantes pesquisados em seu estudo admitiram desconhecer a resposta ou erraram ao serem questionados quanto ao tempo do tratamento prescrito, valor equivalente ao obtido nesta pesquisa, onde $43,81 \%(n=92)$ responderam que não sabiam o tempo da sua terapia medicamentosa, fato semelhante ocorreu quando perguntados sobre o nome do medicamento, como exposto anteriormente (Figura 2).

A Figura 4 traz um paralelo entre o conhecimento dos pacientes acerca da utilização e a conduta em caso de esquecimento. Existe uma grande variedade de medicamentos que possuem características diferentes e formas distintas para serem utilizados, nesse caso, é importante que o usuário saiba quais as particularidades de cada medicamento que ele utiliza como forma de evitar o mau uso. Sabendo disso, dos participantes inclusos na pesquisa, 75,71\% ( $\mathrm{n}=159$ ) conheciam a forma de utilizar o medicamento, sendo essa e a indicação terapêutica as variáveis com menor deficiência de conhecimento presente nesta pesquisa.

Independentemente do valor expressivo, esse resultado é inferior ao encontrado em um estudo anterior, onde demonstrou que a média dos usuários que sabiam como administrar o medicamento foi superior a 95\%, desta forma, é possível afirmar que apesar do índice de conhecimento sobre a forma de administração seja superior aos outros parâmetros encontradas no município, isso não indica níveis satisfatórios de conhecimento, principalmente quando o resultado é comparado à mesma variável em outros estudos (Zanetti et al., 2020).

Ao serem questionados sobre o que fazer em caso de esquecimento, os usuários que responderam que sabiam $(\mathrm{n}=145)$, relataram que a conduta é realizar a medicação no momento que lembrar e/ou no dia seguinte em horário habitual. Apesar desta conduta ser uma solução válida com grande parte dos medicamentos, essa prática deve ser evitada sem o conhecimento adequado, pois há medicamentos que precisam da manutenção regular no organismo devido a sua meia-vida ( $\left.\mathrm{t}_{1 / 2}\right)$ e a necessidade da constância de uma certa concentração no organismo do indivíduo para que não haja comprometimento do tratamento, enquanto outros, podem ser tóxicos quando o intervalo mínimo entre as doses não é respeitado (Esher e Coutinho, 2017; Lima et al., 2017). 
Figura 4 - Comparativo das respostas relacionadas ao conhecimento de como utilizar o medicamento e a conduta em caso de esquecimento dos usuários. Piripiri-PI, 2020.

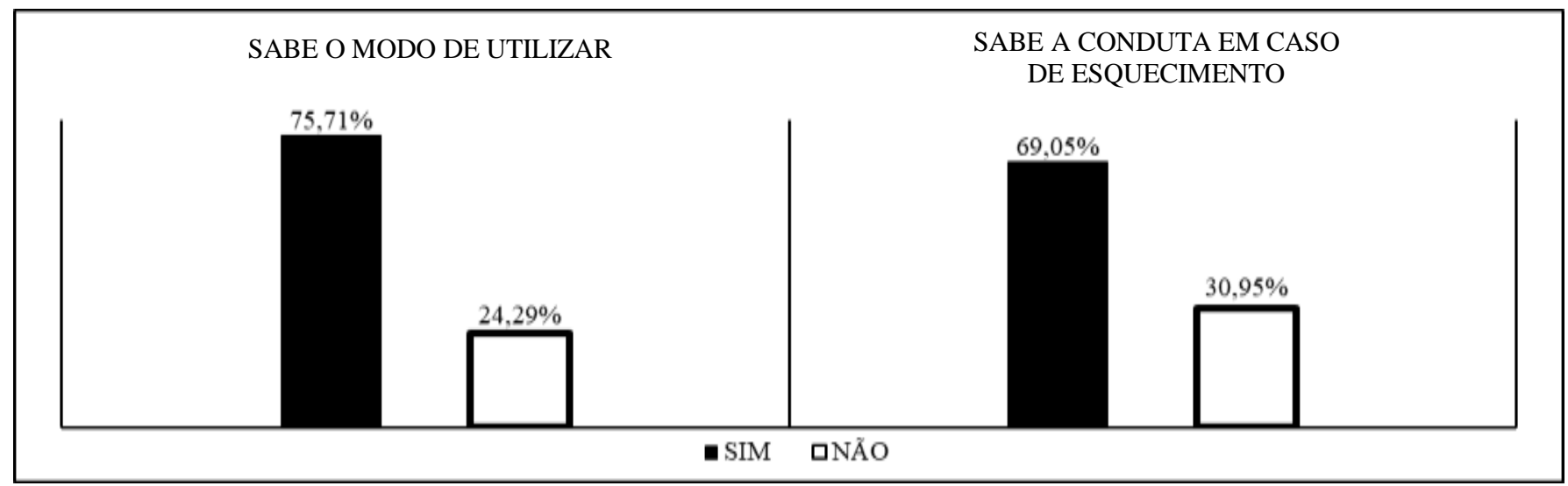

Fonte: Autores (2020).

A variável esquecimento também foi avaliada em outras pesquisas, havendo uma variação significativa entre elas. Dentre as estatísticas pesquisadas na literatura, a menor taxa de conhecimento da conduta em caso de esquecimento foi de $18,9 \%$ a $20,6 \%$, enquanto a mais alta foi de $67,2 \%$, índice semelhante ao encontrado nesta pesquisa (Oliveira, 2018; Zanetti et al., 2020).

A Figura 5 ilustra as respostas obtidas em relação ao conhecimento dos participantes sobre a interação medicamentosa com outros alimentos, bebidas e remédios. Observando o gráfico é visível que a maioria dos usuários $(59,52 \%$ / n=125) não sabem se existem outro produto que ao ser consumido pode provocar alteração no resultado do tratamento e/ou até efeitos adversos. Dos que conheciam sobre interações $(40,8 \%$ / $n=85), 96,43 \%(n=81)$ relataram que o medicamento não poderia ser associado à bebida alcoólica, enquanto apenas 3,57\% $(\mathrm{n}=3)$ informou que ao tomar a medicação não poderia ingerir frituras e outras comidas gordurosas.

Visto isso, é possível observar que todos os participantes identificaram apenas o risco da ingestão de alimentos gordurosos e bebida alcoólica, desconhecendo a relação da interação medicamentosa com outros medicamentos. Esse resultado pode ter relação com a cultura local e o conhecimento empírico da população acerca do uso de medicamentos.

Figura 5 - Comparativo das respostas relacionadas ao conhecimento do usuário acerca da interação medicamentosa. PiripiriPI, 2020.

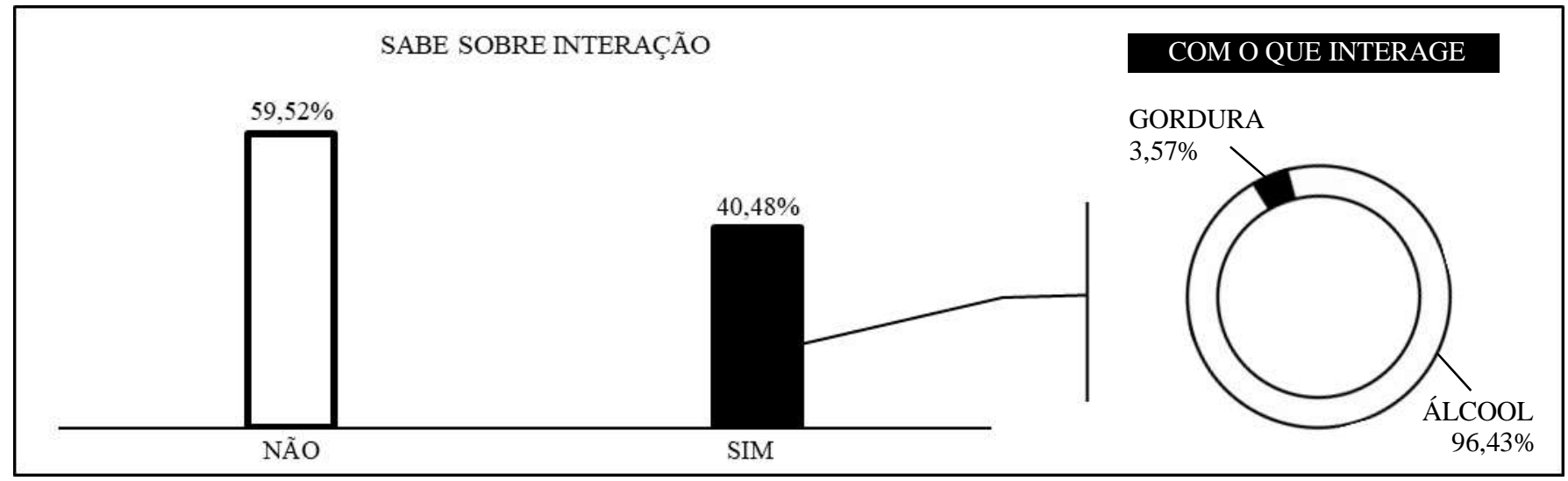

Fonte: Autores (2020). 
Em trabalho realizado no ano de 2018, sobre o nível de conhecimento das prescrições médica entre os usuários da APS de um município de Sergipe, o resultado encontrado referente ao conhecimento da interação medicamentosa foi semelhante a este, onde 39,62\% afirmaram conhecer a existência de bebidas, alimentos e medicamentos que não devem ser ingeridos como forma de evitar interações quando em uso do medicamento prescrito, porém esse número foi reduzindo em outra pesquisa realizada no sul do Brasil (Oliveira, 2018; Dresch et al., 2016).

A Figura 6 representa as respostas obtidas dos participantes relacionadas ao conhecimento acerca da existência de possíveis reações adversas do medicamento. Quando perguntados sobre isso, a maioria dos participantes $(60 \%$ / n=126) não tinham conhecimentos sobre esse tema, resultando na maior taxa de deficiência de conhecimento entre todas as variáveis usadas para avaliar os participantes.

Esse resultado pode ser consequência de consultas que priorizam informações fundamentais para que o usuário adote o tratamento ao invés de instruções sobre fatos que podem interferir de algum modo no desfecho da terapia medicamentosa, não informando e/ou escrevendo na receita (Dresch et al. 2016).

Dos participantes que afirmaram conhecer os possíveis efeitos adversos $(40 \%$ / n=84), 34,52\% ( $\mathrm{n}=29)$ informaram já ter apresentado uma reação, enquanto 65,48\% $(\mathrm{n}=55)$ nunca apresentou nenhuma das reações (Figura 6). Em outra pesquisa, o número de participantes que informaram já ter apresentado alguma reação foi de 37,5\%, próximo do resultado encontrado entre os usuários inclusos nesta pesquisa. No mesmo estudo, a taxa dos que não conheciam sobre efeitos adversos foi de 51,88\%. Fröhlich et al. (2010), encontraram que 74,4\% não sabia dessa informação (Oliveira, 2018).

Figura 6 - Comparativo das respostas relacionadas ao conhecimento do usuário acerca das reações adversas. Piripiri-PI, 2020.

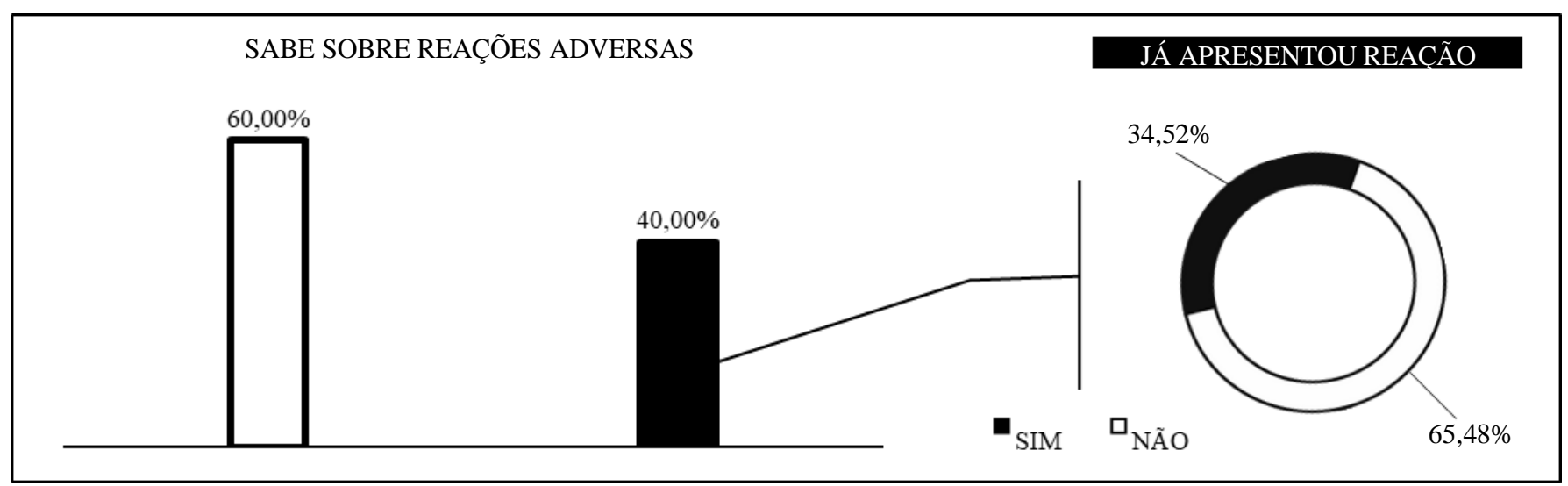

Fonte: Autores (2020).

Em outra pesquisa já citada anteriormente, em média 28,5\% conheciam os efeitos adversos e destes, $53,25 \%$ já tinham manifestado alguma das reações referidas ou sabiam da reação por terem manifestado previamente durante alguma etapa do tratamento. Zanetti et al. (2020), trouxe que poucos participantes sabiam sobre os efeitos adversos do seu medicamento, indicando a carência dessas informações na rotina dos profissionais prescritores para assegurar o URM (Zanetti, 2016).

A próxima ilustração (Figura 7) mostra a necessidade de informações que os usuários mais indicaram precisar e sentem deficiência quanto ao uso do medicamento prescrito, demonstrando que 57\% ( $\mathrm{n}=120)$ dos usuários reconhecem a necessidade de mais orientação para dar continuidade a sua terapêutica de forma adequada. Do número total de participantes, apenas 42,86\% ( $\mathrm{n}=90)$ informaram que não necessitavam de mais informações para utilizar o medicamento de forma adequada.

Segundo Oliveira (2017), dos usuários que informaram não precisar de mais informações foi de 75\%, apesar de 65\% dos participantes apresentarem um nível deficiente de conhecimento sobre a sua prescrição. Resultado semelhante ao 
encontrado por Coradi et al. (2016), onde a maioria dos participantes demonstraram conhecimento deficitário em relação aos pontos necessários para a utilização da terapêutica prescrita.

Figura 7 - Comparativo das respostas relacionadas à necessidade de mais informações acerca do uso de medicamentos dos usuários. Piripiri-PI, 2020.

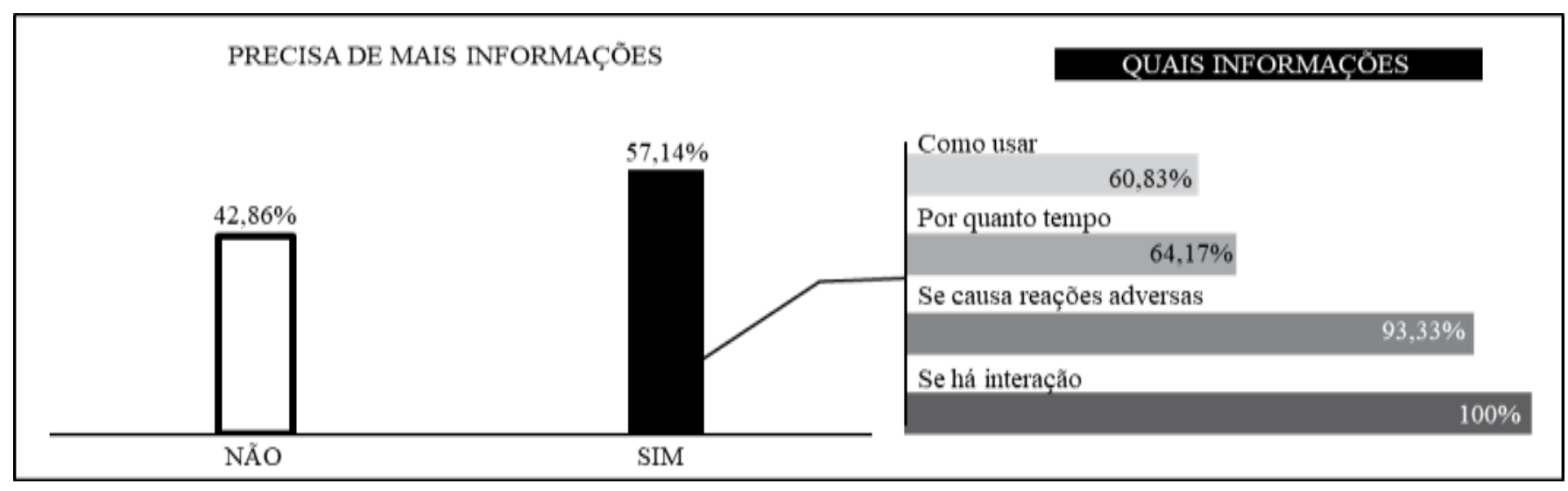

Fonte: Autores (2020).

Das informações que os participantes apontaram necessitar para melhorar a qualidade do processo de medicação, o tocante "Como usar" e "Por quanto tempo" (Figura 7) apresentou a menor taxa de dúvidas, correspondendo ao número de 73 e 77 dos usuários respectivamente. Esse número inferior é proporcional à quantidade de usuários que afirmaram conhecer o modo de usar (Figura 4). Com relação às alternativas "Se causa reações adversas" e "Se há interação", esse número é superior, tendo respectivamente 93,33\% ( $\mathrm{n}=112)$ e 100\% ( $\mathrm{n}=120)$ dos participantes que informaram necessitar de mais informações, confirmando assim, a falta de conhecimento apresentada anteriormente (Figura 5 e 6), sobre as interações medicamentosas e os efeitos adversos.

\section{Conclusão}

O trabalho teve como objetivos avaliar o nível de conhecimento dos usuários da Atenção Primária à Saúde do município de Piripiri-PI acerca das suas prescrições e analisar o perfil sociodemográfico dessa população. Diante disso, podese afirmar que esses objetivos inicialmente propostos foram alcançados com êxito.

Os resultados encontrados mostram que há inúmeras questões deficitárias relacionadas às prescrições de medicamentos que não favorecem o URM na APS. É evidente a necessidade de educar os profissionais prescritores para que eles vejam as prescrições como colaboradoras no tratamento que garante o URM. De similar relevância é a instrução constante dos usuários sobre a sua terapia medicamentosa, promovida pelas equipes de saúde.

Em síntese, diante dos resultados encontrados com relação ao perfil sociodemográfico da população, foi possível verificar que em sua maioria é de baixa renda e baixa escolaridade, fator que tem relação direta com a forma que o paciente compreenderá o que foi prescrito. Enquanto ao nível de conhecimento, conclui-se que há um déficit nesse quesito, principalmente em relação aos efeitos adversos, interações medicamentosas e o tempo do tratamento, favorecendo o uso irracional de medicamentos.

Com relação às possíveis fontes de viés da pesquisa, pode-se destacar que os usuários não foram divididos em grupos relacionados à cronicidade do uso do medicamento, de forma que os participantes que fazem uso prolongado do tratamento podem ter maior conhecimento sobre o que eles fazem uso. Apesar disso, a pesquisa permanece relevante, pois contribui com a compreensão das características e necessidades dos usuários da APS no município. 


\section{Referências}

Aquino, D. S. D. (2008). Por que o uso racional de medicamentos deve ser uma prioridade? Ciência \& Saúde Coletiva, 13, 733-736.

Arrais, P. S. D., Brito, L. L., Barreto, M. L., \& Coelho, H. L. L. (2005). Prevalência e fatores determinantes do consumo de medicamentos no Município de Fortaleza, Ceará, Brasil. Cadernos de Saúde Pública, 21, 1737-1746.

Ascef, B. D. O., Haddad, J. P. A., Álvares, J., Guerra Junior, A. A., Costa, E. A., Acurcio, F. D. A., \& Silveira, M. R. (2017). Qualidade de vida relacionada à saúde dos usuários da atenção primária no Brasil. Revista de Saúde Pública, 51, 22s.

Banõs-Diéz, J. E. \& Farré-Albaladejo, M. (2002). Princípios de Farmacologia Clínica: Aspectos sociológicos del empleo de medicamentos. Barcelona: Masson.

Brasil. Instituto Brasileiro de Geografia e Estatística. (2019b). Cidades IBGE. https://cidades.ibge.gov.br/brasil/pi/piripiri/panorama.

Brasil. Ministério da saúde. (2001a). Política Nacional de Medicamentos. Secretaria de Políticas de Saúde. Departamento de atenção básica. http://www.saude.al.gov.br/wp-content/uploads/2016/04/politica_medicamentos.pdf.

Brasil. Ministério da Saúde. (2019a). Uso de Medicamentos e Medicalização da Vida: recomendações e estratégias. Secretaria de Ciência, Tecnologia e Insumos Estratégicos. Departamento de Assistência Farmacêutica e Insumos Estratégicos. http://bvsms.saude.gov.br/bvs/public acoes/medicamentos_medicalizacao_recomendacoes_estrategia_1ed.pdf.

Brasil. Ministério do Desenvolvimento Agrário. (2015). Perfil Territorial: Cocais - PI. Secretária de Desenvolvimento Territorial. http://sit.mda.gov.br/download/caderno/caderno_territorial_161_Cocais\%20-\%20PI.pdf.

Coradi, C. O., dos Santos Cardoso, J., de Souza Groia, R. C., Silva, K. C. L., Ceccato, M. D. G. B., \& Lima, M. G. (2016). Compreensão da prescrição de medicamentos em uma unidade básica de saúde: autorrelato do usuário e aferição pelo pesquisador. Scientia Medica, $26(4), 7$.

Costa, C. M. F. N., Silveira, M. R., Acurcio, F. D. A., Guerra Junior, A. A., Guibu, I. A., Costa, K. S., \& Álvares, J. (2017). Utilização de medicamento pelos usuários da atenção primária do Sistema Único de Saúde. Revista de Saúde Pública, 51, 18s.

Dresch, A. P., Amador, T. A., \& Heineck, I. (2016). Conhecimento dos pacientes sobre medicamentos prescritos por odontólogos no sul do Brasil. Ciência \& Saúde Coletiva, 21, 475-484.

Esher, A., \& Coutinho, T. (2017). Uso racional de medicamentos, farmaceuticalização e usos do metilfenidato. Ciência \& Saúde Coletiva, 22, 2571-2580.

Fröhlich, S. E., Dal Pizzol, T. D. S., \& Mengue, S. S. (2010). Instrumento para avaliação do nível de conhecimento da prescrição na atenção primária. Revista de Saúde Pública, 44, 1046-1054.

Guibu, I. A., Moraes, J. C. D., Guerra Junior, A. A., Costa, E. A., Acurcio, F. D. A., Costa, K. S., \& Álvares, J. (2017). Carac terísticas principais dos usuários dos serviços de atenção primária à saúde no Brasil. Revista de Saúde Pública, 51, 17s.

Higashi, T., Shekelle, P. G., Solomon, D. H., Knight, E. L., Roth, C., Chang, J. T., \& Wenger, N. S. (2004). The quality of pharmacologic care for vulnerable older patients. Annals of Internal Medicine, 140(9), 714-720.

Lima, M. G., Álvares, J., Guerra Junior, A. A., Costa, E. A., Guibu, I. A., Soeiro, O. M., \& Acurcio, F. D. A. (2017). Indicadores relacionados ao uso racional de medicamentos e seus fatores associados. Revista de Saúde Pública, 51, 23s.

Lyra Junior, D. P., Prado, M. C. T. A., Abriata, J. P., \& Pelá, I. R. (2004). Recetas médicas como causa de riesgo de problemas relacionados con medicamentos. Pharmacy Practice, 2(2), 86-96.

Melo, D. O. D., Ribeiro, E., \& Storpirtis, S. (2006). A importância e a história dos estudos de utilização de medicamentos. Revista Brasileira de Ciências Farmacêuticas, 42(4), 475-485.

Oliveira, G. F. D. (2017). Conhecimento sobre os medicamentos prescritos entre pacientes de uma farmácia comunitária do município de Salgado-SE.

Oliveira, R. D. C. (2018). Nível de conhecimento sobre a prescrição médica entre usuários da atenção básica de Lagarto/SE.

Opas. Organização Pan-Americana De Saúde. (2005). Avaliação da Assistência Farmacêutica no Brasil: estrutura, processo e resultados. https://tinyurl.com/y8sy8649.

Opas. Organização Pan-Americana De Saúde. (2007). Uso racional de medicamentos na perspectiva multiprofissional. Ministério da Saúde. https://tinyurl.com/y9ez5693.

Portela, A. D. S., Simões, M. O. D. S., Fook, S. M. L., Montenegro Neto, A. N., \& Silva, P. C. D. D. (2010). Prescrição médica: orientações adequadas para o uso de medicamentos? Ciência \& Saúde Coletiva, 15, 3523-3528.

Scripcaru, G., Mateus, C., \& Nunes, C. (2017). Adverse drug events-Analysis of a decade. A Portuguese case-study, from 2004 to 2013 using hospital database. PloS one, 12(6), e0178626.

Sharif, S. I., Al-Shaqra, M., Hajjar, H., Shamout, A., \& Wess, L. (2008). Patterns of drug prescribing in a hospital in Dubai, United Arab Emirates. Libyan Journal of Medicine, 3(1), 10-12.

Souza, T. F. D., Colet, C. F., \& Heineck, I. (2018). Nível de informação e adesão à terapia de anticoagulação oral com varfarina em pacientes acompanhados em ambulatório de atenção primária à saúde. Jornal Vascular Brasileiro, 17(2), 109-116. 
Research, Society and Development, v. 10, n. 5, e2610514487, 2021

(CC BY 4.0) | ISSN 2525-3409 | DOI: http://dx.doi.org/10.33448/rsd-v10i5.14487

Who. World Health Organization et al. (1986). The rational use of drugs. https://apps.who.int/medicinedocs/documents/s16221e/s16221e.pdf.

Zanetti, M. O. B. (2016). Prescrição de medicamentos e compreensão do paciente na Atenção Primária à Saúde: análise comparativa entre o modelo de atendimento básico tradicional e a Estratégia de Saúde da Família no município de Ribeirão Preto-SP (Dissertação de Mestrado). Universidade de São Paulo, São Paulo, SP, Brasil. Recuperado de https://teses.usp.br/teses/disponiveis/60/60137/tde-30012017-082429/en.php.

Zanetti, M. O. B., Marchetti, J. M., \& de Andrade, R. C. G. (2020). Compreensão do usuário da Atenção Primária à Saúde sobre o tratamento farmacológico: uma análise comparativa. Journal of Applied Pharmaceutical Scienses, (7), 1-10. 